\title{
Small reductions in risk factors equal big savings in health-care costs
}

A $1 \%$ decrease in the incidence of cardiovascular events across the population of England and Wales could produce annual financial savings of at least $£ 30$ million. An economic model constructed by Barton and colleagues also indicates that costs could be reduced to an even greater extent if mean blood pressure (BP) or cholesterol levels were reduced by just $5 \%$ in this population.

This model was built to guide a publichealth program set up by the UK National Institute for Health and Clinical Excellence. The researchers aimed to determine the potential savings in terms of quality of life years (QALYs) and health-care costs of risk-factor reduction programs, specifically $\mathrm{BP}$ and cholesterol lowering. By reducing the average UK daily salt intake by $3 \mathrm{~g}$ per day from the current level of $8.5 \mathrm{~g}$ per day, the model predicted a mean reduction in $\mathrm{BP}$ of $2.5 \mathrm{mmHg}$, equating to a $2 \%$ risk reduction in the population. Such an intervention would prevent 32,200 cases of cardiovascular disease and 4,430 cardiac deaths over a 10 -year period, saving $£ 40$ million per year and gaining 131,000 QALYs. Similarly, a 5\% reduction in mean cholesterol level would prevent 64,000 cases of cardiovascular disease and 8,800 deaths over 10 years, saving $£ 80$ million per year and gaining 260,000 QALYs. Research conducted in other countries indicates that these reductions could be exceeded and, therefore, the estimated cost savings are likely to be conservative.

The authors conclude that "any intervention that achieved even a modest population-wide reduction in any major cardiovascular risk factor would produce a net cost saving ... as well as improving health."

Alexandra King

Original article Barton, P. et al. Effectiveness and cost effectiveness of cardiovascular disease prevention in whole populations: modelling study. BMJ 343, d4044 (2011) 